RBGF- Revista Brasileira de Geografia Física

Recife-PE, Vol.2 n.2, maio-agosto 2009, 11-18

\title{
UM ESTUDO PRELIMINAR DE POSSÍVEIS EFEITOS DE MUDANÇAS CLIMÁTICAS NO NORDESTE DO BRASIL
}

\author{
José Maria Brabo Alves, \\ Maryfrance de Cássia Santos Diniz ${ }^{2}$
}

Artigo recebido em 21/08/2009 e aceito para publicação em 31/08/2009.

\begin{abstract}
RESUMO
Possíveis evidências de mudanças climáticas globais no Nordeste do Brasil (NEB) são investigadas entre os períodos 1950-1970 e 1980-2000; 1974-1990 e 1991-2003, os primeiros quase sem influência antropogênica e os dois últimos períodos com possível influência antropogênica. Foi usada uma série de precipitação diária (1974-2003) observada em pontos de grade e dados mensais de precipitação e temperatura do ar (1950-2000) das reanálises do National Center for Environment Predicition (NCEP)-National Center for Atmospheric Research (NCAR) admitindo ser dados representativos da variabilidade de grande escala climática na Região. Os resultados mostraram um aumento nos totais anuais de precipitação (15\% a 25\%) e na temperatura média anual (até $0,6^{\circ} \mathrm{C}$ ) sobre o NEB no período 1981-2000 comparado ao período 1950-1970. Estatísticas de extremos (número de dias com chuva forte $>10 \mathrm{~mm}$ e com chuva leve $<=3 \mathrm{~mm}$ considerado sem precipitação), em uma área média representativa do setor norte do NEB durante fevereiro a maio, indicaram uma diminuição no período 1991-2003 em relação ao período 1974-1990.
\end{abstract}

Palavras Chaves: mudança climática, variabilidade interanual, Nordeste do Brasil.

\section{A PRELIMINARY STUDY OF POSSIBLE EFFECT OF CLIMATIC CHANGES NORTH-EASTERN OF BRAZIL}

\begin{abstract}
Possible evidences of global climate change in northeastern Brazil (NEB) are investigated from periods 1950-1970 and 1980-2000, 1974-1990 and 1991-2003, first almost without antropogênica influence and the two last periods with possible antropogênica influence. A observed daily precipitation (1974-2003) series was used in the grid points on the and monthly data of precipitation and air temperature (1950-2000) reanalysis of the National Center for Environment Prediction (NCEP) - National Center for Atmospheric Research (NCAR) assuming data are representative of large-scale climate variability in the region. The results showed an increase in total annual precipitation (15\% to $25 \%$ ) and average annual temperature (up to $0.6^{\circ} \mathrm{C}$ ) on the NEB in the period 1981-2000 compared to the period 1950-1970. Statistics of extremes (number of days with heavy rain $>10 \mathrm{~mm}$, with light rain $<=3 \mathrm{~mm}$ considered without precipitation) in a mean area representing the northern sector of NEB during February-May, showed a decrease in the period 1991-2003 for the period 1974-1990.
\end{abstract}

Key-words: Climate change, interannual variability, Northeast Brazil.

\footnotetext{
${ }^{1}$ Meteorologista, Dr. em Engenharia Civil - Recursos Hídricos, FUNCEME-CE, e-mail: brabo@funceme.br

${ }^{2}$ Mestre em Meteorologia, INGÁ-BA, e-mail: mary_france@hotmail..com
} 


\section{RBGF- Revista Brasileira de Geografia Física}

Recife-PE, Vol.2 n.2, maio-agosto 2009, 11-18

\section{INTRODUÇÃO}

No Brasil, dependendo do cenário de emissão de gases na atmosfera, os aumentos de temperatura são estimados em torno de 1 a $6{ }^{\circ} \mathrm{C}$, o que deverá aumentar a evaporação à superfície e por sua vez alterações no balanço hídrico da vegetação natural e das culturas agrícolas (NOBRE, 2001). Esse efeito deve ser mais negativo em regiões onde predominam a agricultura de sequeiro; a não ser que o aumento de temperatura seja acompanhado de aumento e/ou regularização no regime de chuvas.

O Nordeste do Brasil (NEB) atravessa ao longo dos anos por uma variabilidade natural do clima, alternando anos de chuvas extremas a secas severas (NOBRE et al. 1996; MOURA \& SHUKLA, 1991; ALVES et al. 2003; SUN et al. 2005 e outros) que associada à ação antropogênica pode mudar totalmente as características do clima de uma determinada região.

Evidências globais têm mostrado variações climáticas ao longo de precipitação e uma tendência positiva de aumento de temperatura relacionada com o aquecimento global (GEMMER et al. 2004; ENDO et al. 2005; QIN et al. 2005, DANG et al. 2007). A questão de mudanças interdecadais de características climáticas desérticas a semidesérticas tem sido muito observada na Ásia, nos desertos de Taklamakan e Gobi (KIM et al. 2008). Na região do semiárido do Nordeste brasileiro, KROL \& BRONSTERT (2007) identificaram tendência significativa de redução nas vazões do Rio Jaguaribe após 2025, considerando cenário de redução de $50 \%$ da precipitação nas próximas cinco décadas. Para um cenário de redução de 21\% da precipitação, estes autores não encontraram tendência significativa de alteração da vazão.

Este estudo pretende investigar uma possível influência da ação do homem (antropogênica) no NEB usando precipitação e temperatura, duas principais variáveis meteorológicas consideradas componentes primárias do clima que refletem, inicialmente, ao efeito de mudanças climáticas (KÖPPEN, 1931, QIN et al. 2005). Perguntas por que estão mais freqüentes nestes últimos 16 anos aproximadamente extremos de chuvas, como número de dias secos $(<2 \mathrm{~mm})$ ou com chuvas consideradas fortes $>10 \mathrm{~mm}$ no NEB, comparado ao período 1974-1990 (“com menor influência antropogênica”), poderão ser respondidas.

\section{MATERIAL E MÉTODO}

Neste artigo foram utilizados dados de reanálises de precipitação e temperatura do ar próximo à superfície obtidos do National Center for Environment Prediction (NCEP) - 


\section{RBGF- Revista Brasileira de Geografia Física}

Recife-PE, Vol.2 n.2, maio-agosto 2009, 11-18

National Center for Atmospheric Research (NCAR) para o período de 1950-2000 selecionados em uma área que compreende $90^{\circ} \mathrm{W}-10^{\circ} \mathrm{E}$ e $60^{\circ} \mathrm{S}-60^{\circ} \mathrm{N}$, aproximadamente. Os resultados podem não representar a variabilidade intra-regional no NEB, portanto, devem ser interpretados com cautela devido à baixa densidade espacial de informações destas variáveis sobre a região que alimenta as reanálises. Dados diários de precipitação entre 1974-2003 medidos em pluviômetros distribuídos no Nordeste do Brasil (Figura 1) também foram usados neste estudo. As fontes destes dados são do Instituto Nacional de Meteorologia (INMET), Agência Nacional de Energia Elétrica (ANNEL), Agência Nacional de Águas (ANA) e Núcleos Estaduais de Meteorologia e Recursos Hídricos do NEB. Detalhes sobre o processo de controle e qualidade destes dados podem ser obtidos em Souza et al. (2004). São médias diárias calculadas entre os dias 28 e 29 nos fevereiros bissextos. Complementando estes dados foram usados também totais anuais de produtividade de milho e feijão para o estado do Ceará (1974-2004), obtidos no Instituto de Planejamento do Estado do Ceará (IPLANCE).

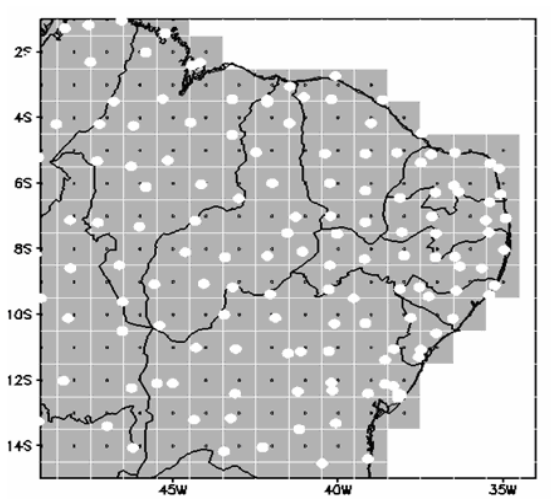

Figura 1: Postos pluviométricos com observações diárias (pontos em branco) de precipitação usada no estudo. Os pontos em preto indicam o centro da grade de interpolação dos dados.

Inicialmente, a partir dos dados de reanálises foram determinadas as anomalias em relação ao total anual de chuva e a temperatura média anual entre os períodos 1951-1970 e 1990-1990. A significância destas anomalias foi calculada segundo Harrison et al (1998). Em seguida, usando os dados de precipitação diária observada no NEB, foram estimados o número de dias secos $(<=2 \mathrm{~mm})$, chuvosos $(>2 \mathrm{~mm})$, com chuva leve $(<=3 \mathrm{~mm})$ e com chuva intensa $(>10 \mathrm{~mm})$ para os períodos 19741990 e 1991-2003. Esta última estimativa foi feita para a faixa entre $55^{\circ} \mathrm{W}-34^{\circ} \mathrm{W}$ e $12^{\circ} \mathrm{S}-2^{\circ} \mathrm{S}$ sobre o NEB, onde juntamente com os dados de produtividade de milho e feijão plotou-se histogramas com distribuição normal aproximada. Para esta mesma área e com o número de dias considerados secos e com chuva intensa, foram plotados gráficos de 


\section{RBGF- Revista Brasileira de Geografia Física}

Recife-PE, Vol.2 n.2, maio-agosto 2009, 11-18

distribuição de freqüências acumuladas para alguns anos de contrastes climáticos classificados como de El Niño, La Niña e Normais entre o período de 1974-2003 (ALVES et al. 2007).

\section{RESULTADOS E DISCUSSÕES}

A Figura 2 mostra a anomalia de precipitação (totais anuais) e temperatura média anual sobre o NEB, resultante das diferenças entre os períodos de 1981-2000 e 1950-1970. Nota-se que tanto para precipitação como para temperatura houve um aumento. Para a precipitação (Figura 2a) este aumento no período de 1981-2000 em grande parte do NEB, foi da ordem de $10 \%$ a 30\% em relação ao período de 1950-1970. Este aumento de precipitação é consistente com os estudos de Rao et al. (2006) que mostraram um incremento de chuvas no NEB nestes últimos anos, principalmente após os anos 70. Para a temperatura (figura 2b) foi observado um aquecimento de até $0,6^{\circ} \mathrm{C}$ na região semi-árida do $\mathrm{NEB}$, consistente com a expectativa do IPCC e estudos mais recentes como os de Jones et al.(2007).
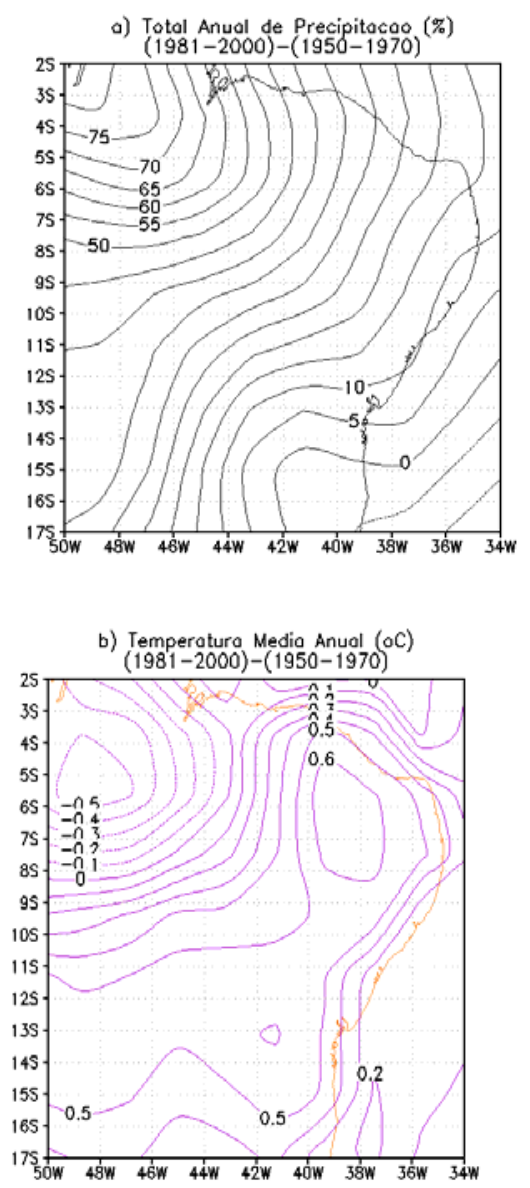

Figura 2: Anomalias em relação ao período de 1950-1970 obtidas pela diferença (1981-2000)(1950-1970): a) totais anuais de precipitação e b) temperatura média anual.

A Figura 3 apresenta o número de dias $\operatorname{secos}(<=2 \mathrm{~mm})$ e chuvosos $(>2 \mathrm{~mm})$ ao longo do NEB para os períodos 1974-1990 e 1991-2003. A diferença mais marcante nestas configurações é uma expansão em área no sentido nordeste, do número de dias secos no período 1991-2003 e uma pequena diminuição do número de dias chuvosos neste mesmo período em comparação ao período 1974-1990. 


\section{RBGF- Revista Brasileira de Geografia Física}

Recife-PE, Vol.2 n.2, maio-agosto 2009, 11-18

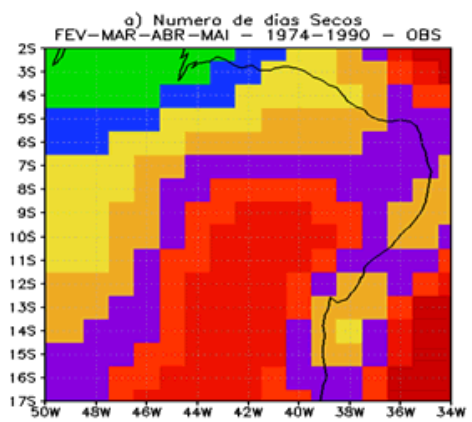

para o período FEV-MAR-ABR-MAI referente ao número de dias com chuva leve ( $<=3 \mathrm{~mm})$ e chuva definida como forte ( $>10 \mathrm{~mm}$ ) para uma área média $\left(12^{\circ} \mathrm{S}-2^{\circ} \mathrm{S}\right.$ e $\left.55^{\circ} \mathrm{W}-34^{\circ} \mathrm{W}\right)$ no $\mathrm{NEB}$ representativa do semi-árido. Em ambas as situações são notáveis uma redução tanto do número de dias com chuva leve quanto com chuva intensa (inferior a 12 dias), principalmente, no período 1991-2003. Estes resultados, em particular, para chuvas definidas intensas são similares aos encontrados por Marengo \& Ambrizzi (2006).
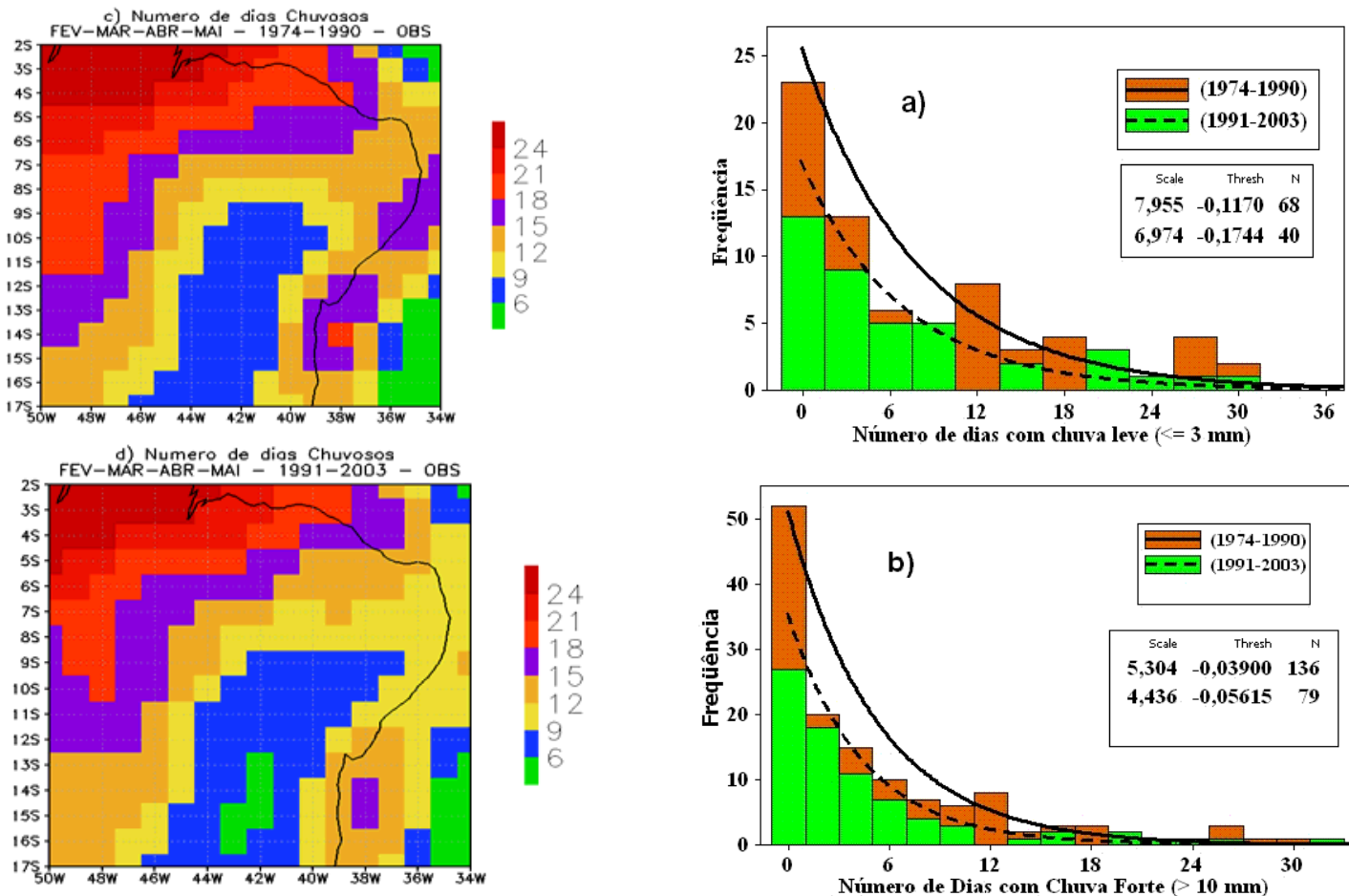

Figura 3: Climatologia para: a) e b) número de dias secos e c) e d) número de dias chuvosos entre os meses de fevereiro e maio nos períodos de 1974-1990 e 1991-2003.

A Figura 4 exibe um histograma com aproximação de uma função exponencial

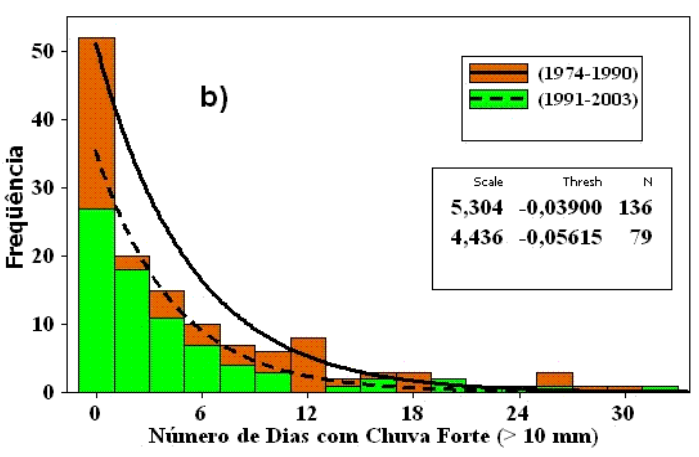

Figura 4: Histograma do número de dias com chuva leve ( $<=3 \mathrm{~mm}$ - fig. 4a) e chuva forte ( $>$ $10 \mathrm{~mm}$ - fig. 4b) entre os meses de fevereiro e maio no setor norte do NEB. 


\section{RBGF- Revista Brasileira de Geografia Física}

Recife-PE, Vol.2 n.2, maio-agosto 2009, 11-18

No período 1974-2003 e nos anos classificados como de El Niño, La Niña e Normais, as distribuições de freqüências acumuladas nos meses de FEV-MAR-ABRMAI indicam algumas características interessantes (Figura 5). Para precipitações leves, nos anos de La Niña as probabilidades em média superam o clima, e nos anos de El Niño e Normais essas probabilidades são inferiores para todo o espectro do número de dias. O inverso ocorre no caso do número de dias com chuva forte, ou seja, nos anos de El Niño e Normais às probabilidades são superiores ao clima (entre cinco e dez dias), enquanto para os anos de La Niña estas são inferiores ao clima (entre zero e dez dias).
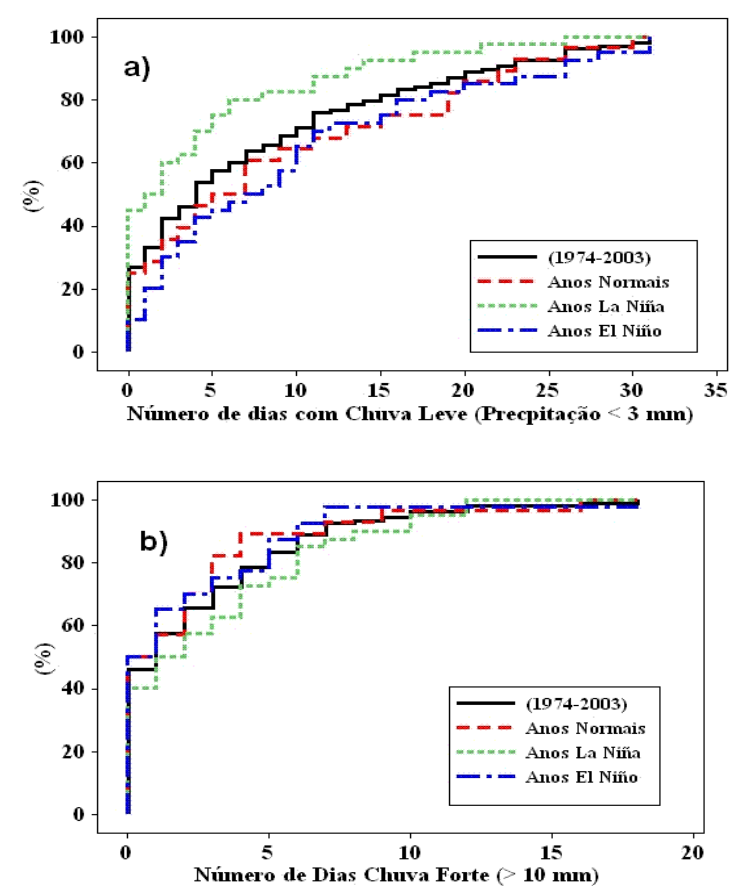

Figura 5: Distribuição de probabilidade acumulada do número de dias com chuva leve $(<3 \mathrm{~mm}-$ fig. $5 \mathrm{a})$ e chuva forte $(>10$ mm - fig. 5b) para o período 1974-2003, em anos de El Niño, La Niña e Normais durante os meses entre fevereiro e maio no setor norte do NEB.

\section{CONCLUSÃO E SUGESTÃO}

Apesar da amostra de anos ser pequena para conclusões mais categóricas, dentre as principais conclusões mostradas no estudo citase: i) espacialmente no NEB, com os dados de reanálises, ocorreu um aumento em torno de 10 \% a 30\% do total anual de precipitação, bem como, um aquecimento da ordem de até $0.6^{\circ} \mathrm{C}$ na temperatura média anual no período 19812000 em relação ao período 1950-1970; ii) com os dados observados de precipitação para o período 1991-2003, foi verificada uma expansão no número de dias definidos como $\operatorname{secos}(<=2 \mathrm{~mm})$ e uma pequena diminuição no número de dias chuvosos ( $>2 \mathrm{~mm}$ ) para o setor nordeste da Região, possivelmente relacionados há uma maior influência antropogênica ao comparar com o período 1974-1990; iii) na média, para uma região semi-árida do NEB selecionada $\left(12^{\circ} \mathrm{S}-2^{\circ} \mathrm{S}\right.$ $55^{\circ} \mathrm{W}-34^{\circ} \mathrm{W}$ ), foi observada uma redução do número de dias com chuvas leves $(<=3 \mathrm{~mm})$ e fortes (> 10mm) no período de 1991-2003. Recomenda-se que em estudos futuros façam comparações destes resultados com os cenários do IPCC. 


\section{RBGF- Revista Brasileira de Geografia Física}

Recife-PE, Vol.2 n.2, maio-agosto 2009, 11-18

\section{REFERÊNCIAS}

ALVES, J. M. B.; CAMPOS, J. N. B.; NASCIMENTO, L. S. V. \& BARROS, F. V. F. Estudo preliminar intrasazonal do acoplamento entre modelagem dinâmica de precipitação e vazão no Nordeste do Brasil. In: Anais do XVII Simpósio Brasileiro de Recursos Hídricos e $8^{\circ}$ Simpósio de Hidráulica e Recursos Hídricos de Países de Língua Oficial Portuguesa. São Paulo, Novembro. 2007.

ALVES, J. M. B. et. al. Uma aplicação da técnica de downscaling dinâmico no setor norte da Região Nordeste do Brasil. Rev. Bras. Meteor., v.18, n.2, p.161-180, 2003.

DANG, H.; GILBERT, N. P.; WEAVER, A. J. and ZWIERS, E. W. Climate change detection over different land surface vegetation classes. Int. J. Climatol., 27, p.211-220. 2007.

ENDO, N.; AILIKUN, B. and YASUNARY, T. Trends in precipitation amounts and the number of rainy days and heavy rain events during summer in China from 1961 to 2000. J. Meteoro. Soc. Japan, 83, p.621-331. 2005.

GEMMER, M.; BECKER, S. and JIANG, T. Observed monthly precipitation trends in China 1951-2002. Theor. Appl. Climatol., 77, p.39-45. 2004.

HARRISON, D. E.; LARKIN, N. K. El Niño-Southern Oscillation sea surface temperature and wind anomalies, 1946-1993. Rev. Geophysics, v. 36, n.3, p. 353-399. 1998.

JONES R.G.; MURPHY, J.M.; HASSEL, D.C., WOODAGE, M.J. A high resolution atmospheric GCM for the generation of regional climate scenarios. Climate Dynamics in prelo. 2007.

KIN, H-J.; WANG, B.; DING, Q. and CHUNG, U-H. Changes in arid climate over north China detected by the Köppen climate classification. J. Meteoro. Soc. Japan, 86, p.981-990. 2008.

KÖPPEN, W. Grundriss der Klimakunde, Walter de Gruyter, 388pp. 1931.

KROL, M.S.; BRONSTERT, A. Regional integrated modelling of climate change impacts on natural resources and resource usage in semi-arid Northeast Brazil. Environmental Modelling \& Software, Oxford, v.22, p.25968, 2007.

KROL, M.; JAEGER, A.; BRONSTERT, A.; GÜNTNER, A. Integrated modelling of climate, water, soil, agricultural and socioeconomic processes: A general introduction of the methodology and some exemplary results from the semi-arid north-east of Brazil. Journal of Hydrology, Amsterdam, v.328, p.417-31, 2006.

MARENGO, J., AMBRIZZI, T. Use of regional climate models in impacts assessments and adaptations studies from continental to regional and local scales: The CREAS (Regional Climate Change Scenarios for South America) initiative in South America. Proceedings of 8 ICSHMO, Foz do Iguaçu, Brazil, April 24-28, 2006, p.291-296. 2006.

MOURA, A. D.; SHUKLA, J. On the dynamics of droughts in northeast Brazil: Observations, theory and numerical experiments with a general circulation model. J. Atmos. Scie., v.38, n.12, p.2653-2675. 1981. 
NOBRE, C.A. Mudanças climáticas globais: possíveis impactos nos ecossistemas do País. Parcerias Estratégicas, Brasília, n.12, p.239-58, 2001.

NOBRE, P.; SHUKLA, J. Variations of sea surface temperatures, wind stress, and rainfall over the tropical over the tropical Atlantic and South America. J. Climate., v.9, n.10, p.2464-

QIN, D. H.; DING, Y. H.; SU, J. L. and WANG, S. M. Climate and Environment Changes in China. China Science Press, 562p. 2005.

RAO, V. B.; GIAROLLA, M.; KAYANO, M. T.; FRANCHITO, S. H. Is the recent increasing trend of rainfall over Northeast Brazil related to Sub-Saharan drought? J. Climate, v.19, p.4448-4453. 2006.

SOUZA, E. B.; KAYANO, M. T. \& AMBRIZZI, T. The regional precipitation over eastern amazon/northeast Brazil modulated by tropical Pacific and Atlantic SST anomalies on weekly timescale. Rev. Bras. Meteor., v.19, n.2, p.113-122. 2004.

SUN, L.; MONCUNILL, D. F.; LI, H.; MOURA, A. D.; FILHO, F. D. D. S. Climate downscaling over Nordeste Brazil using NCEP RSM97. J. Climate,18, p.551-567. 2005. 